\title{
Endoscopic treatment for iatrogenic achalasia post-laparoscopic adjustable gastric banding
}

\author{
Radu Pescarus ${ }^{1} \cdot$ Ahmed Sharata $^{2} \cdot$ Eran Shlomovitz $^{1} \cdot$ Kevin Reavis $^{2}$. \\ ${\text { Christy } \text { Dunst }^{2} \cdot \text { Lee Swanstrom }}^{2,3}$ (D)
}

Received: 27 May 2015/Accepted: 9 September 2015/Published online: 30 October 2015

(C) Springer Science+Business Media New York 2015

\begin{abstract}
Background Esophageal obstruction is a known complication of laparoscopic adjustable gastric band (LAGB) and usually occurs in the context of band slippage. Current reports of pseudoachalasia post-LAGB describe that in some patients esophageal function improves after band removal. For those without improvement, current treatments include division of the fibrotic band post-LAGB or revisional surgery. Our hypothesis, illustrated in the submitted video, is that an endoscopic division of LAGB-induced stricture will improve esophageal function.

Methods This video presents the case of a patient presenting with dysphagia post-LAGB removal. The preoperative high-resolution manometry was compatible with a type I achalasia. Using a high-definition endoscope and the equipment used for per-oral endoscopic myotomy (POEM) procedure, the intramural fibrotic tissue caused by the LAGB is divided, thus releasing the stricture and restoring baseline esophageal function. This is demonstrated with intraoperative endoscopic functional lumen imaging probe (EndoFLIP; Crospon Ltd, Galway, Ireland).

Results Similar to the POEM technique, a 12-cm tunnel and 8 -cm myotomy were performed. No intra-procedural or post-procedural complications were noted. Using
\end{abstract}

impedance planimetry, the division of the LAGB-induced stricture induced an increase in the minimal diameter from 5.3 to $8.6 \mathrm{~mm}$. The cross-sectional area increased from 22 to $58 \mathrm{~mm}^{2}$. The patient denies any residual dysphagia, regurgitation, or heartburn at 6-month follow-up. On the postoperative high-resolution manometry, significant improvement in LES pressure parameters was seen postmyotomy with return of $30 \%$ peristalsis.

Discussion In LAGB patients with pseudoachalasia in which removal of fluid from the band does not result in clinical or manometric improvement, laparoscopic removal of the band with division of the peri-esophageal scar tissue has been advocated. This video illustrates that an endoscopic division of the LAGB-induced esophageal fibrosis is another potential treatment for adjustable gastric band-induced achalasia.

Keywords Gastric banding · Iatrogenic achalasia . POEM

\section{Compliance with ethical standards}

Disclosures Drs. Pescarus, Sharata, Shlomovitz, Reavis, Dunst, and Swanstrom have no conflicts of interest or financial ties to disclose.
Electronic supplementary material The online version of this article (doi:10.1007/s00464-015-4561-7) contains supplementary material, which is available to authorized users.

\footnotetext{
Lee Swanstrom

lswanstrom@gmail.com

Providence Portland Cancer Center, Portland, OR, USA

2 The Oregon Clinic, Portland, OR, USA

3 IHU-Strasbourg, Strasbourg, France
} 